\title{
ICU admission rates in Istanbul following the addition of favipiravir to the national COVID-19 treatment protocol
}

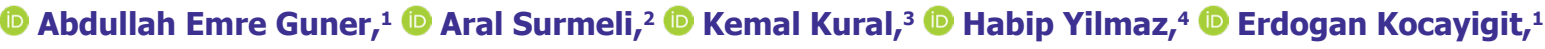 \\ (D) Esra Sahin, ${ }^{1}$ (D) Perihan Alkan, ${ }^{5}$ (D) Cemal Kazezoglu, ${ }^{6}$ (D) Serdal Zelyurt, ${ }^{7}$ (D) Kemal Memisoglu, ${ }^{8}$ \\ Isil Maral9 \\ ${ }^{1}$ Istanbul Health Directorate, Public Health Services, Istanbul, Turkey \\ ${ }^{2}$ Medical Rescue Association of Turkey, Istanbul, Turkey \\ ${ }^{3}$ Istanbul Health Directorate, Monitoring, Evaluation and Statistics, Istanbul, Turkey \\ ${ }^{4}$ Istanbul Health Directorate, Department of Public Hospital Services, Istanbul, Turkey \\ 5Istanbul Health Directorate, Infectious Diseases, Istanbul, Turkey \\ ${ }^{6}$ Health Sciences University, Kanuni Sultan Suleyman Training and Research Hospital, Istanbul, Turkey \\ ${ }^{7}$ Istanbul Health Directorate, Support Services, Istanbul, Turkey \\ ${ }^{8}$ Istanbul Health Directorate, Director of Health, Istanbul, Turkey \\ ${ }^{9}$ Department of Public Health, Istanbul Medeniyet University Faculty of Medicine, Istanbul, Turkey
}

\begin{abstract}
OBJECTIVE: The objective of this study was to understand the observational relationship between adoption of favipiravir into the national COVID-19 treatment protocol and intensive care unit (ICU) admission rates in Istanbul due to COVID-19.

METHODS: Data were harvested from the "Public Health Management System-HSYS," which collate centrally the records of all known cases of COVID-19. The total number of cases, numbers admitted to ICU, and number undergoing intubation were compared between 2 time periods: $11^{\text {th }}$ of March, the date on which the first case in Turkey was confirmed, to $30^{\text {th }}$ of March; and March 30, to $10^{\text {th }}$ of April, 5 days after Favipiravir was introduced into the treatment algorithm when, the records were examined.
\end{abstract}

RESULTS: The percentage of patients requiring ICU admission diminished from $24 \%$ to $12 \%$, whilst the percentage intubated fell from $77 \%$ to $66 \%$. These differences were both statistically significant.

CONCLUSION: The addition of favipiravir to the national COVID-19 treatment protocol may explain this rapid decrease in the rate of ICU admissions and intubation.

Keywords: COVID-19; favipiravir; intensive care unit.

Cite this article as: Guner $A E$, Surmeli $A$, Kural K, Yilmaz H, Kocayigit E, Sahin E, et al. ICU admission rates in Istanbul following the addition of favipiravir to the national COVID-19 treatment protocol. North Clin Istanb 2021;8(2):119-123.

$\mathrm{T}$ he pandemic that started in December 2019 in the city of Wuhan, China, has since spread all around the globe, resulting in more than 6.5 million cases and
380,000 fatalities as of the end of May $2020[1,2]$. Turkey has recorded that 166,422 cases of COVID-19 confirmed by polymerase chain reaction (PCR) and

Received: November 10, 2020 Accepted: February 01, 2021 Online: March 22, 2021

Correspondence: Aral SURMELI, MD. Turkiye Medikal Arama Kurtarma Dernegi, Istanbul, Turkey.

Tel: +90 5353140179 e-mail: aralsurmeli@hotmail.com

(c) Copyright 2021 by Istanbul Provincial Directorate of Health - Available online at www.northclinist.com 
4609 deaths [3]. Even though the number of new cases has been steadily decreasing and the rate of fatalities has also been relatively low ( 55 deaths/million), the disease burden is still high, and it remains possible that a second peak of infections may occur.

Turkey implemented the National Pandemic Preparedness Plan and instigated preparatory measures in early January, at the time when the initial reports of COVID-19 from Wuhan appeared. Stockpiling of Personal Protective Equipment (PPE) and various medications was undertaken in the initial phase, whilst public health measures such as school closures and the cancellation of international flights to high-risk regions were soon implemented [4]. In addition, the National COVID-19 Treatment Protocol was drawn up at an early stage and has since undergone regular updating by the Turkish Ministry of Health and COVID Scientific Committee [5].

Favipiravir is an antiviral pharmaceutical agent originally developed in Japan to combat influenza infection [6]. It is currently one of several agents being evaluated in the treatment of COVID-19 [7]. Whilst the evidence base at present remains limited, preliminary results from various trials appear to show efficacy in decreasing the need for intensive care unit (ICU) admission and shortening the length of stay due to COVID-19 [8]. The Turkish National COVID-19 Treatment Protocol incorporated favipiravir into its algorithm from the updated version of $25^{\text {th }}$ of March onward. Simultaneously, distribution and use of the drug began following the delivery of 1 million units from China [9]. The dosage recommendations for Favipiravir were as follows: $600 \mathrm{mg}$ daily for 5 days following a single loading dose of 1600 $\mathrm{mg}$ on the $1^{\text {st }}$ day. The drug was provided without cost to the patient at the point of care. The treatment algorithm also calls for concomitant use of hydroxychloroquine and oseltamivir for cases admitted to ICU. Pre-ICU cases in Istanbul were also administered favipiravir.

The present study is an ecological study comparing the rates of ICU admission and intubation before the inclusion of favipiravir in the National COVID-19 Treatment Protocol with those in the immediate aftermath of its inclusion.

\section{MATERIALS AND METHODS}

Ethical board approval for the study was granted by the Istanbul Medeniyet University Ethical Board on $23^{\text {rd }}$ of April 2020 with decree number 2020/0202. Anonymized data were extracted from national

\section{Highlight key points}

- ICU admitted and intubated patients were of older age (median 61 and 56 respectively).

- There seems to be a dramatic change in ICU admission rates after addition of Favipiravir to the treatment regimen.

- Favipiravir should be included in controlled and randomized study protocols for treatment of severe COVID-19 cases.

COVID-19 surveillance system for analysis. Every hospital in Turkey inputs demographic and clinical data for all suspected and confirmed cases of COVID-19 on a daily basis. No consent was needed form individual patients as only existing, aggregated data from the national database were used. The Turkish Ministry of Health's case definition for COVID-19, wherein laboratory confirmation of SARS-CoV-2 is necessary, which was used. The retrieved data were then separated into two groups, relating to the period before $30^{\text {th }}$ of March and after $30^{\text {th }}$ March up to 5 days before the analysis (April 10) [10]. Only inpatients were counted, since favipiravir was only given to inpatients, either in ICU or pre-ICU. The choice of $30^{\text {th }}$ of March was due to the inclusion of favipiravir in the National COVID-19 Treatment Protocol on $25^{\text {th }}$ of March, following a 5-day course of treatment.

\section{Statistical Analysis}

The cumulative number of hospital admissions, ICU admissions, and intubation were calculated using the $\mathrm{Mi}$ crosoft Excel (2019, Microsoft, New Mexico/ABD) application and analyzed in SPSS (2020, IBM, New York/ $\mathrm{ABD})[11,12]$.

The age and sex distribution of the groups are presented separately. Age grouping was done into 10 yearintervals. For cases whose age was below 19 years, the data are presented in aggregate, due to the low number of such cases. The percentage of cases undergoing intubation is calculated by taking the overall number of cases admitted to ICU as the denominator since intubation was only performed on cases admitted to ICU.

The difference in two proportions test (z-score) was used to analyze the difference among specific age and sex groups between both timeframes. When the numbers of cases were too low, Fischer's Exact test was used, and for age groups below a sample size of 10, P-value was not calculated. The P-value is presented for each group unless the number of cases was too low. P-value below 0.05 was taken to indicate statistical significance. P-values indicat- 


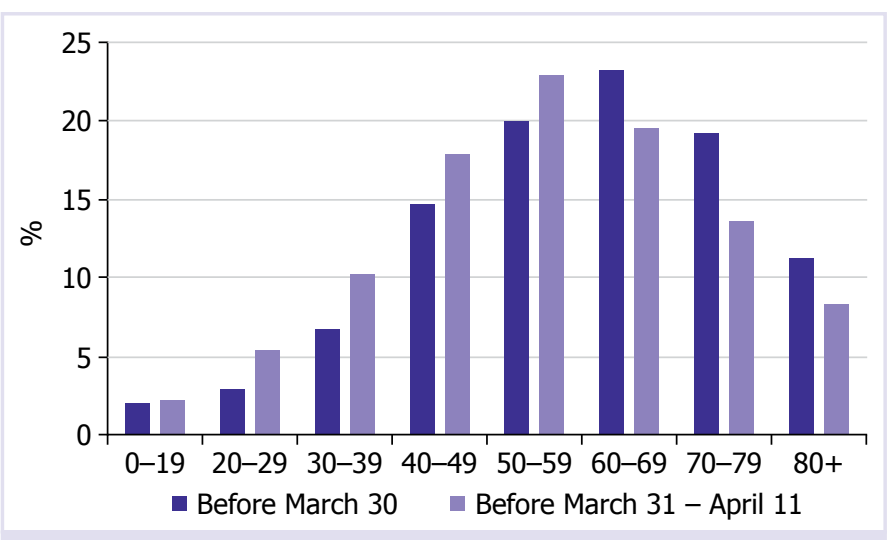

FIGURE 1. Age distribution in the between two groups.

ing statistical significance are bolded in table. No extra power calculation was undertaken as sampling included the entire patient population at that time.

\section{RESULTS}

The median age in the group who was hospitalized before March $30^{\text {th }}$ was 61 years (IQR: 49-72) and the group between March $31^{\text {st }}$ and April $10^{\text {th }}$ was 56 (IQR: 44-68). The age group distribution is presented in Figure 1 . The cases aged below 19 and over 80 were aggregated due to the low numbers of cases in both groups. Sex distribution was $65 \%$ male, $35 \%$ female in the group before March 30 and 55\% male, and 45\% female in the group between March 31 and April 10.

From the first confirmed case of COVID-19 in Istanbul on March 11, 2020 until March 30, 2020, there were 3301 confirmed cases of COVID-19 necessitating hospital admission. From that point until April 10, 2020, 7060 further cases of COVID-19 were confirmed by PCR testing and subsequently admitted. In the first period, 802 cases $(24 \%)$ were admitted to ICU, of which $617(77 \%)$ were intubated. In the second period, 817 cases $(12 \%)$ were admitted to ICU, while 543 (66\%) of those underwent intubation. Age and sex groups of ICU admitted and intubated patients are presented in Table 1. Percentages of intubation are only from ICU admitted patients, as no intubation was done outside of ICU.

ICU admission rates were significantly lower in all age groups with the exception of patients aged over 80 $(p=0.4691)$. Intubation rates among ICU patients differed significantly; however, only if the patients were aged above 60. No P-value is provided either due to low number of events or similar percentage levels in intubated patients in the table.

\section{DISCUSSION}

This is a descriptive, ecological study about the difference in rate of ICU admission and intubation between two groups, which differed in terms of treatment regimen, one receiving favipiravir, the other not. There are statistically significant differences in the rates of ICU admissions and intubation needs between the two groups. Five days after favipiravir began to be used nationwide, the rates of ICU admission and intubation rates fell in Istanbul. Even though primary reports on the use of favipiravir appear promising, there remains a lack of robust evidence to favor the use of this agent [13].

In the study population, which covers all the $\operatorname{PCR}(+)$ COVID-19 patients in Istanbul, the percentage of cases admitted to ICU fell from $24 \%$ to $12 \%$, while the intubation rate among ICU patients decreased from $77 \%$ to $66 \%$. We hypothesize that favipiravir may have played a role in decreasing ICU admission and intubation rates. Among all age groups, other than in patients aged over 80, ICU admission rates fell significantly. This difference in intubation rates is less significant when age groups are individually examined. The median age was 5 years younger in the second group and the prognosis of COVID-19 appears to be more favorable in younger age groups [14]. In addition, the sex distribution changed in favor of females in the second group. There is evidence that COVID-19 has a worse prognosis in male patients [15].

Despite the possibility that confounders may have influenced the result to a certain extent, the effect of favipiravir integration was deemed clinically to have been swift and effective. Accordingly, favipiravir was distributed to hospitals; the same day, it was added to the protocol in Istanbul and was rapidly used by clinicians. A decision had already been put in place whereby all treatment for COVID-19 is free at the point of care to all patients. At present, Turkey has not surpassed its healthcare, ICU bed, or intubation capacity needs at any point, meaning virtually all patients requiring testing or treatment have been able to receive it without encountering any economical or health-care capacity barriers.

In a rapidly evolving pandemic, the medical response changes frequently with emerging data and evidence. There is a need for more controlled, randomized, and rigorous studies to be done in order to understand the actual relationship and effect of favipiravir on prognosis of confirmed COVID-19 patients. This study indi- 
TABLE 1. ICU admission and intubation rates in Istanbul [1]

\begin{tabular}{|c|c|c|c|c|c|c|c|c|c|}
\hline & \multicolumn{4}{|c|}{ Admitted to ICU } & \multirow[b]{3}{*}{$\mathrm{p}$} & \multicolumn{4}{|c|}{ Intubated } \\
\hline & \multicolumn{2}{|c|}{ Before March 30} & \multicolumn{2}{|c|}{$\begin{array}{l}\text { Between March } 31 \\
\text { and April } 10\end{array}$} & & \multicolumn{2}{|c|}{ Before March 30} & \multicolumn{2}{|c|}{$\begin{array}{l}\text { Between March } 31 \\
\text { and April } 10\end{array}$} \\
\hline & Count & Percentage & Count & Percentage & & Count & Percentage & Count & Percentage \\
\hline \multicolumn{10}{|l|}{ All } \\
\hline 0-19 & 26 & 39 & 17 & 11 & 0.0001 & 12 & 46 & 6 & 35 \\
\hline $20-29$ & 11 & 11 & 18 & 5 & 0.015 & 8 & 73 & 12 & 67 \\
\hline $30-39$ & 36 & 16 & 32 & 4 & 0.0001 & 27 & 75 & 21 & 66 \\
\hline $40-49$ & 78 & 16 & 78 & 6 & 0.0001 & 57 & 73 & 46 & 59 \\
\hline $50-59$ & 158 & 24 & 129 & 8 & 0.0001 & 115 & 73 & 91 & 71 \\
\hline $60-69$ & 212 & 28 & 177 & 13 & 0.0001 & 174 & 82 & 124 & 70 \\
\hline $70-79$ & 176 & 28 & 212 & 22 & 0.0064 & 140 & 80 & 143 & 67 \\
\hline $80+$ & 105 & 28 & 154 & 26 & 0.4691 & 84 & 80 & 100 & 65 \\
\hline Total & 802 & 24 & 817 & 12 & 0.0001 & 617 & 77 & 543 & 66 \\
\hline \multicolumn{10}{|l|}{ Male } \\
\hline 0-19 & 15 & 38 & 8 & 11 & 0.0007 & 5 & 33 & 3 & 38 \\
\hline $20-29$ & 8 & 13 & 12 & 7 & 0.1494 & 7 & 88 & 10 & 83 \\
\hline $30-39$ & 28 & 18 & 23 & 5 & 0.0001 & 20 & 71 & 14 & 61 \\
\hline $40-49$ & 54 & 16 & 62 & 8 & 0.0001 & 40 & 74 & 35 & 56 \\
\hline $50-59$ & 116 & 25 & 96 & 10 & 0.0001 & 85 & 73 & 66 & 68 \\
\hline $60-69$ & 155 & 30 & 118 & 16 & 0.0001 & 125 & 81 & 77 & 65 \\
\hline 70-79 & 113 & 30 & 115 & 23 & 0.019 & 89 & 79 & 80 & 70 \\
\hline $80+$ & 59 & 32 & 68 & 26 & 0.1658 & 49 & 83 & 43 & 63 \\
\hline Total & 548 & 26 & 502 & 13 & 0.0001 & 420 & 77 & 328 & 65 \\
\hline \multicolumn{10}{|l|}{ Female } \\
\hline 0-19 & 11 & 39 & 9 & 11 & 0.0009 & 7 & 64 & 3 & 30 \\
\hline $20-29$ & 3 & 8 & 6 & 3 & 0.1435 & 1 & 33 & 2 & 33 \\
\hline $30-39$ & 8 & 13 & 9 & 3 & 0.0001 & 7 & 88 & 7 & 78 \\
\hline $40-49$ & 24 & 16 & 16 & 3 & 0.8936 & 17 & 71 & 11 & 69 \\
\hline 50-59 & 42 & 22 & 33 & 5 & 0.0001 & 30 & 71 & 25 & 76 \\
\hline 60-69 & 57 & 22 & 59 & 9 & 0.0001 & 49 & 86 & 47 & 80 \\
\hline $70-79$ & 63 & 25 & 97 & 21 & 0.1981 & 51 & 81 & 63 & 65 \\
\hline $80+$ & 46 & 25 & 86 & 26 & 0.8042 & 35 & 76 & 57 & 66 \\
\hline Total & 254 & 22 & 315 & 10 & 0.0001 & 197 & 78 & 215 & 68 \\
\hline
\end{tabular}

Percentages of intubation are only from ICU admitted patients, as no intubation was done outside of ICU. ICU: Intensive care unit.

cates that the use of favipiravir in COVID-19 could be a promising area for future research.

There are several limitations and strengths of this study. First of all, this is carried out as an ecological study design, where the focus is on understanding the phenomenon, rather than inferring or a directional causality. By nature of this methodology, there could be con- founders such as comorbidity, increased awareness of clinicans about the pandemic, leading to earlier detection of cases, increased experience, and evidence on care for COVID-19 patients. Attributing the ICU admission rates solely on favipiravir cannot be deduced.

The strength of this study is using the whole population, each patient who was diagnosed with COVID-19 
and had to receive in hospital care in Istanbul, where majority of the cases of COVID-19 were at the time in Turkey. In addition, the timeframes are short and consequtive, meaning the changing characteristic of the disease such as decreasing average age of severe cases in coming months, should have a minimal effect. However, given the limitations within the evidence base and within this study, we cannot unequivocally propose clinical use of favipiravir or change of treatment algorithms in favor of favipiravir. There is an urgent need for more robust and controlled clinical trials with addition of favipiravir to the research protocol.

The opinions expressed by authors contributing to this article do not necessarily reflect the opinions of the Ministry of Health, Istanbul Health Directorate or the institutions with which the authors are affiliated.

Acknowledgements: We would like to thank Pelin Emiroglu, MD; Ipek Akdeniz, MD; Alparslan Duman, MD; Savas Bayrak, MD for their support for this manuscript. Finally, we acknowledge the tremendous work done by healthcare workers around the world, who are risking their lives daily to take care of their patients.

Ethics Committee Approval: The Istanbul Medeniyet University Clinical Research Ethics Committee granted approval for this study (date: 22.04.2020, number: 2020/0202).

Conflict of Interest: No conflict of interest was declared by the authors.

Financial Disclosure: The authors declared that this study has received no financial support.

Authorship Contributions: Concept - AEG, AS; Design - IM, AEG; Supervision - IM, KM, CK; Fundings - AS, ES, KK; Materials - HY, EK, CK; Data collection and/or processing - SZ, EK; Analysis and/or interpretation - HY, PA; Literature review - CK, PA; Writing - AS, KK, SZ; Critical review - EK, ES, IM.

\section{REFERENCES}

1. Guan WJ, Ni ZY, Hu Y, Liang WH, Ou CQ, He JX, et al; China Medical Treatment Expert Group for Covid-19. Clinical characteristics of coronavirus disease 2019 in China. N Engl J Med 2020;382:1708-20.
2. Coronavirus Update (Live): $6,513,890$ cases and 384,642 deaths from COVID-19 virus pandemic - Worldometer (Internet). Available at: https://www.worldometers.info/coronavirus/. Accessed Jun 3, 2020 .

3. COVID-19 - Yeni koronavirüs hastalığ (new coronavirus disease) (Internet). Available at: https://covid19bilgi.saglik.gov.tr/tr. Accessed Jun 2, 2020 .

4. Pandemik influenza ulusal hazırlık planı (National pandemic influenza preparedness plan). Ankara: Ministry of Health of Turkey, Public Health Directorate; 2019. Available at: https://hsgm.saglik.gov.tr/tr/ bulasicihastaliklar-haberler/ulusal-pandemi-hazirlik-plani.html. Accessed May 25, 2020.

5. Algoritmalar C. COVID-19 Algoritmalar (COVID-19 Treatment Protocol- Version 1). Available at: https://covid19bilgi.saglik.gov.tr/ tr/algoritmalar. Accessed Jan 30, 2020.

6. Furuta Y, Komeno T, Nakamura T. Favipiravir (T-705), a broad spectrum inhibitor of viral RNA polymerase. Proc Jpn Acad Ser B Phys Biol Sci 2017;93:449-63. [CrossRef]

7. Lu CC, Chen MY, Lee WS, Chang YL. Potential therapeutic agents against COVID-19: What we know so far. J Chin Med Assoc 2020;83:534-6. [CrossRef]

8. Dong L, Hu S, Gao J. Discovering drugs to treat coronavirus disease 2019 (COVID-19). Drug Discov Ther 2020;14:58-60. [CrossRef]

9. Algoritmalar C. COVID-19 Algoritmalar (COVID-19 Treatment Protocol- Version 6). Available at: https://covid19bilgi.saglik.gov.tr/ tr/algoritmalar. Accessed Mar 25, 2020.

10. World Health Organization. Surveillance, rapid response teams, and case investigation. Available at: https://www.who.int/emergencies/diseases/novel-coronavirus-2019/technical-guidance/surveillance-andcase-definitions. Accessed May 18, 2020.

11. Microsoft Corporation. Microsoft Excel (Internet). 2018. Available at: https://office.microsoft.com/excel. Accessed Feb 24, 2021.

12. IBM Corp. IBM SPSS Statistics for Windows (Internet). Armonk, NY: IBM Corp; 2017. Available at: https://hadoop.apache.org. Accessed Feb 24, 2021.

13. Cai Q, Yang M, Liu D, Chen J, Shu D, Xia J, et al. Experimental treatment with favipiravir for COVID-19: an open-label control study. Engineering (Beijing) 2020;6:1192-8. [CrossRef]

14. Grasselli G, Zangrillo A, Zanella A, Antonelli M, Cabrini L, Castelli A, et al; COVID-19 Lombardy ICU Network. Baseline characteristics and outcomes of 1591 patients infected with SARSCoV-2 admitted to ICUs of the Lombardy region, Italy. JAMA 2020;323:1574-81. [CrossRef]

15. Zheng Z, Peng F, Xu B, Zhao J, Liu H, Peng J, et al. Risk factors of critical $\&$ mortal COVID-19 cases: A systematic literature review and meta-analysis. J Infect 2020;81:e16-25. [CrossRef] 